\title{
Low Anterior Resection of Rectal Cancer and Vaginal Specimen Extraction with Da Vinci Xi
}

\author{
Tuğrul Çakır, Arif Aslaner \\ University of Health Sciences, Antalya Training and Research Hospital, Department of \\ General Surgery, Antalya, TURKEY
}

\begin{abstract}
:
Introduction: Novel robotic surgery systems (da Vinci Xi) are superior to classical open and laparoscopic techniques with its clear and threedimensional view. We aimed to present the first case low anterior resection of rectal cancer and vaginal specimen extraction with Da Vinci Xi.
\end{abstract}

Case: A 75-year-old female patient with rectum adenocarcinoma was undergone robotic-assisted low anterior resection (LAR) of the rectum, vaginal removal of the specimen, colorectal anastomosis and loop ileostomy. The operation time was 190 minutes. There were no postoperative complications. Pathological tumor stage was stage pT1N0 with negative proximal, distal and radial resection margins. The patient was discharged on the third postoperative day.

Conclusion: Robot-assisted LAR, total mesorectal excision, vaginal removal of the specimen, colorectal anastomosis, and loop ileostomy can be performed easily and safely with $\mathrm{Da}$ Vinci $\mathrm{Xi}$ at early stage rectal cancer. And the vaginal extraction of the specimen avoids us from a traditional abdominal incision.

Keywords: robotic surgery, da Vinci Xi, rectal cancer, vaginal extraction

\section{Introduction}

Rectal surgery with robotic systems is become more popular, and the da Vinci $\mathrm{Xi}{ }^{\circledR}$ is the latest surgical platform developed by Intuitive Surgical. The better postoperative comfort and cosmesis were provided by natural orifice specimen extraction. A combination of this robotic procedure with vaginal extraction of the specimen can potentiate the benefits of minimally invasive surgery. Here, we presented the first case of low anterior resection of rectal cancer and vaginal specimen extraction with the latest robotic surgical system Da Vinci Xi.

\section{Case Presentation}

On November 25, 2016, a 75-year-old female patient was admitted to our hospital complaining about rectal hemorrhage till the last three months without significant weight loss. After taking her history and physical examination routine laboratory examinations were taken. Abdominal physical examination produced unremarkable findings. There was not any significant past or family history of malignancy of the colon to the patient. The patient's hematological and biochemical findings on admission included; white blood cell

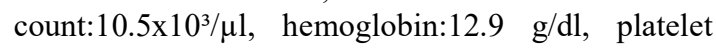
count:357 $\times 10^{3} / \mu \mathrm{l}$, creatinine: $1.09 \mathrm{mg} / \mathrm{dl}$, albumin:3.7 gr/dl, total bilirubin: $0.7 \mathrm{mg} / \mathrm{dl}$, direct bilirubin: $0.13 \mathrm{mg} / \mathrm{dl}$, liver enzymes including SGOT of $16 \mathrm{U} / 1$ (normal range $<50$ ), SGPT of $10 \mathrm{U} / 1$ (normal range $<50$ ), amylase of $40 \mathrm{U} / 1$ (normal range 22-80) and LDH $230 \mathrm{U} / 1$ (normal range <248), INR 0.99, CEA $3.13 \mathrm{ng} / \mathrm{ml}$ (normal range: $<5 \mathrm{ng} / \mathrm{ml}$ ), CA 19-9 $33.8 \mathrm{U} / \mathrm{ml}$ (normal range: $<35 \mathrm{U} / \mathrm{mL}$ ), CA 15-3 43.9 $\mathrm{U} / \mathrm{ml}$ (normal range: $<31 \mathrm{U} / \mathrm{mL}$ ). Her hematologic and biochemical profile including liver function tests and tumor markers were normal. Abdominal ultrasound revealed as normal without grade 2 hepatosteatosis and cholecystectomy. At the rectal digital examination, a mass was palpated at the 7 th $\mathrm{cm}$ away from the dentate line. And the patient underwent to colonoscopy. At the colonoscopic examination, some diverticulum at the sigmoid colon and a mass was seen and biopsies were performed. Biopsy revealed as adenocarcinoma of the rectum. Abdominopelvic Magnetic Resonance Imaging (MRI) through the rectum and abdomen a suspicious increase was seen at the wall of the rectum and multiple diverticula at the sigmoid colon region.

The patient demanded the surgical removal and we offered robotic resection and natural orifice specimen extraction if feasible to the patient. Exact details of the procedure were explained and a patient informed consent was obtained. She had no other surgical history except laparoscopic cholecystectomy. 
Surgical Procedure:

She received mechanical bowel preparation and a single dose of prophylactic antibiotic (Cefuroxime Axetil 1g) 1 hour before the skin incision and antithrombotic prophylaxis was administered with low-molecular-weight heparin 12 hours before. A urinary catheter was inserted.

The procedure was performed using the da Vinci Surgical System Xi (Intuitive Surgical, Sunnyvale, CA, USA). We used Maryland fenestrated bipolar forceps, tip-up double fenestrated grasper, and monopolar scissors.

The operation was performed in two phases, abdominal and pelvic phases. In the abdominal phase, after giving general anesthesia, the patient was placed in a modified dorso-lithotomic position, with a $26^{\circ}$ Trendelenburg position during the procedure. The Veress needle was inserted at the $10 \mathrm{~cm}$ lateral the umbilical level to insufflate the abdomen. After pneumoperitoneum was obtained, an $8 \mathrm{~mm}$ incision was made and the trocar was placed through the incision and three other 8-mm lateral ports were placed under direct visualization. An additional $10 \mathrm{~mm}$ trocar for the assistant is placed in the right upper quadrant. Pneumoperitoneum was kept at $8-12 \mathrm{mmHg}$. The robotic surgical system was docked using fours arms. After induction of pneumoperitoneum and insertion of the $30^{\circ}$ robotic camera and placing all the instruments, the routine whole abdominal cavity exploration was performed. First medial-to-lateral dissection, ligation of the inferior mesenteric artery was performed at the root of the inferior mesenteric artery, than splenic flexure, the sigmoid and descending colon was mobilized to medially. Then rectal mobilization down to the pelvic floor was performed to achieve TME. In this LAR case, the division of the distal rectum was performed using two laparoscopic Endo Gia linear staplers (green cartridge, $60 \mathrm{~mm}$, Ethicon EndoSurgery, Inc., Cincinnati, OH, USA).

In the pelvic phase, after placing a vaginal speculum, an ovarian clamp was stand to the posterior fornix of the vagina. The posterior fornix of the vagina was opened intracorporeally with the electrocautery scissors over the clamp. Then the distal side of the specimen catch with clamp and pull throughout the vagina. (Figure 1) The specimen was divided $15 \mathrm{~cm}$ proximal of the tumor with electrocautery (Figure 2) and the anvil of the circular stapler was put inside the colon and fixed with purse string $2 / 0$ prolene suture. After this procedure, the colon gets inside to the abdomen. A sponge was pushed through the vagina to the posterior fornix. After the digital rectal examination circular stapler $(31 \mathrm{~mm})$ got inside the rectum. With the robotic assistance, the anvil and the stapler were got into together. After the squeezing and firing of the stapler, the colorectal anastomoses were performed. Finally, a diverting ileostomy was established at the right lower side of the abdomen. (Figure 3)
Following placement of a vaginal tampon and an abdominal drain to the pelvis, abdominal trocar site incisions were closed with $3 / 0$ prolene sutures. The total operation time was 190 minutes with a blood loss of fewer than $100 \mathrm{ml}$. There were no postoperative complications. The patient was started on an oral diet postoperative first day and the abdominal drain was removed on the second day. She was discharged from hospital on the third postoperative day.

Histopathology was reported as adenocarcinoma of the rectum with the histologic grade 2 and the pathological stage of the tumor was pT1 with none metastatic lymphatic nodes and negative proximal, distal and radial margins. The ileostomy was closed 2 months after the first operation. She was well after 20 months of treatment and has no signs of the $\mathrm{d}$ isease at the follow-up.

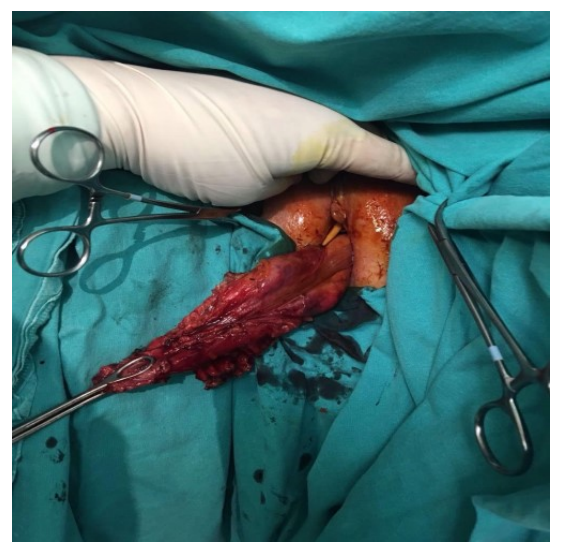

Fig-1: Pulling the distal side of the specimen with clamp throughout the vagina.

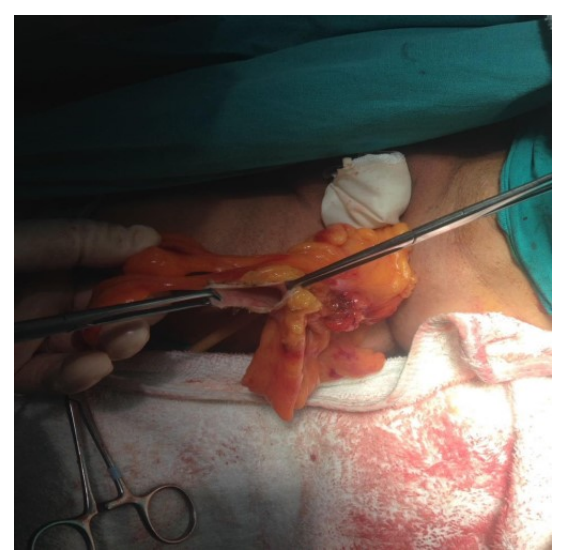

Fig-2: After resection of the tumor proximal segment was seen. 


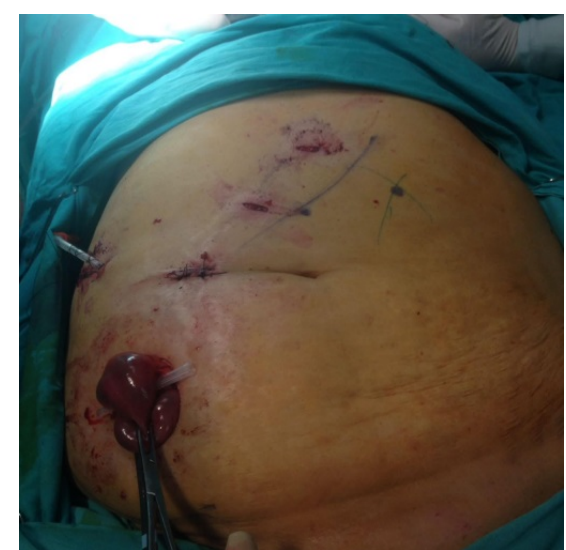

Fig-3: Ileostomy was established at the right lower side of the abdomen

\section{Discussion}

At the year 2001, the practice of laparoscopic vaginal specimen extraction was introduced.1 Here, we report the first case of low anterior resection of rectal cancer and vaginal specimen extraction with the latest robotic surgical system Da Vinci Xi. To our knowledge, this was not reported before.

Natural orifice specimen extraction in laparoscopic surgery avoids extra-abdominal incisions and can ensure less wound-related complications.2 There are three potential natural orifices for laparoscopic surgery: mouth, anus, and vagina. Last two were commonly used for the colorectal specimen extractions.3 Transvaginal extraction, although limited to female patients, can allow the extraction of larger specimens. 2 No size limit has yet been established for transvaginal specimen extraction and removal of a $9 \mathrm{~cm}$ in size right hemicolectomy specimen had been demonstrated before. 4

Transvaginal specimen extraction limitations include the narrow vagina, large masses, virginity, and patient acceptance. High demand of aesthetics renders transvaginal specimen extraction a highly attractive option among young adults. 5 Our results of mean operation time and estimated blood loss were similar with the literature.6,7

In conclusion, LAR for rectal cancer can be performed successfully with da Vinci $\mathrm{Xi}$ and the specimen was extracted through a natural orifice; vagina with satisfying short-term outcomes.

\section{References}

1. Tsin DA, Colombero LT, Mahmood D, Padouvas J, Manolas P: Operative culdolaparoscopy: A new approach combining operative culdoscopy and minilaparoscopy. J Am Assoc Gynecol Laparosc 8, 438-441 (2001) [PubMed]

2. Ersan V, Kayaalp C, Aktas A, Gundogan E, Teomete U, Sumer F, Karagul S. Transvaginal extraction of laparoscopic liver resection specimen.
Interv Med Appl Sci. 2017 Dec;9(4):215-217. doi: 10.1556/1646.9.2017.39. PubMed PMID: 29951289;PubMed Central PMCID: PMC6016203.

3. Kayaalp C, Yagci MA: Laparoscopic right colon resection with transvaginal extraction: A systematic review of 90 cases. Surg Laparosc Endosc Percutan Tech 25, 384-391 (2015) [PubMed]

4. Yagci MA, Kayaalp C, Novruzov NH: Intracorporeal mesenteric division of the colon can make the specimen more suitable for natural orifice extraction. J Laparoendosc Adv Surg Tech A 24, 484-486 (2014) [PubMed]

5. Yagci MA, Kayaalp C: Transvaginal appendectomy: A systematic review. Minim Invasive Surg 2014, 384706 (2014) [PMC free article] [PubMed]

6. Lee HJ, Choi GS, Park JS, Park SY, Kim HJ, Woo IT, Park IK. A novel robotic right colectomy for colon cancer via the suprapubic approach using the da Vinci Xi system: initial clinical experience. Ann Surg Treat Res. 2018 Feb;94(2):83-87. doi: 10.4174/astr.2018.94.2.83. Epub 2018 Jan 30. PubMed PMID: 29441337; PubMed Central PMCID: PMC5801332.

7. Choi GS, Park IJ, Kang BM, Lim KH, Jun SH. A novel approach of robotic-assisted anterior resection with transanal or transvaginal retrieval of the specimen for colorectal cancer. Surg Endosc. 2009 Dec;23(12):2831-5. doi:10.1007/s00464-0090484-5. Epub 2009 May 14. PubMed PMID: 19440794. 